\title{
Video Capture Service in the Intelligent Transportation System based on Cloud Computing
}

\author{
Fekri M. Abduljalil \\ Dept, of Computer Science, \\ Faculty of Educ., Arts, and \\ Science \\ University of Sana'a
}

\begin{abstract}
Vehicular networking has significant potential to enable many different applications related to traffic safety and traffic efficiency within Intelligent Transportation System. In this paper, cloud-based vehicular network architecture is proposed such that the vehicles can share computation resources, storage resources and bandwidth resources. The proposed architecture includes four types of cloud: a vehicular cloud, a roadside cloud, a cellular cloud and a central cloud. Then, a video capture service based on the proposed architecture is presented. Vehicular cloud is used to provide a video capture service of specific location, moving vehicle or route. It sends the captured video to a customer and the other available cloud to be used for further investigation. Resource allocation and management algorithms are proposed to handle the continuity of video capture with minimum service disruption. The proposed service is implemented using a test bed.
\end{abstract}

\section{General Terms}

Computer Networks, Vehicular Networks.

\section{Keywords}

Vehicular Network, Cloud Computing, Video Capture.

\section{INTRODUCTION}

Intelligent Transportation System (ITS) is the utilization of information technology to enhance the transportation system. Elements within the transportation system such as vehicles, roads, traffic lights, and message signs, become smart devices. ITS enables various users to be more coordinated, better informed and smarter use of transport networks. Intelligent transportation System (ITS) is the integration of information system applications and communications technologies to improve transportation safety and mobility and enhance system productivity. Currently, there are many countries have Intelligent Transport Systems such as USA [1], Europe [2], Japan [3], Canada [4] and others.

Nowadays, vehicles become more and more important in our daily life and most people go to work by vehicles, so it is expected that every person will have a vehicle in near future. Since every vehicle can be equipped with a different wireless transceiver, vehicles on the road may form a wireless network named as a vehicular ad hoc network (VANET) and can be integrated with infrastructure wireless communication system such as GPRS/3G, Wi-Fi, WiMAX and LTE[5]. The VANET is a subclass of the mobile ad hoc network (MANET) that it also has no fixed topology. Vehicles may acquire information and services through the V2V (Vehicle-to-Vehicle) and I2V (Infrastructure-to-Vehicle) or R2V (Roadside-to-Vehicle) communications. The $\mathrm{V} 2 \mathrm{~V}$ communication is based on the Dedicated Short Range Communications (DSRC) technology; while the $\mathrm{I} 2 \mathrm{~V}$ communication is based on GPRS/3G, WiFi or WiMAX $[6,7,8,9,10]$. The emergence of new wireless systems, ITS application and services, and ITS technologies, has raised novel research questions on safety and non-safety related applications[11].

Cloud computing is gaining more and more popularity recently. It can be applied to the ITS applications to provide efficient real time services as well as to improve transportation safety, mobility and comfort degree for drivers. The motivation toward cloud computing in vehicular network is the communication, storage and computing resources available in the vehicular networks represented by communication, vehicles, roadside units (RSUs), and central servers. These resources can be utilized and provided to vehicles, drivers on the road, travellers, and customers on the internet. In the transportation context, the cloud computing is faced many challenges such as the production of unified architectural framework and many different services based on mobile computing $[12,13,14]$.

The current research of Research and Innovative Technology Administration (RITA) [1] focuses on real-time data capture and management. Real-time data capture and management is the access to high-quality real-time multi-modal transportation data that is captured from connected vehicles and infrastructure. Such a service promises a wide range of applications in surveillance, traffic monitoring, roads monitoring, and forensics investigation. Because of the huge potential in applications, video capture is considered in our research work.

In this paper, a cloud-based architecture for vehicular networks is proposed. Our work is different from previous researches in that we proposed new cloud based vehicular networks with four types of cloud. A novel service called video capture service is proposed based on cloud computing and the proposed architecture. A new approach for handoff algorithm is proposed to decrease the disruption time when handing service from vehicle to another or from cloud to another cloud.

The remainder of the paper is organized as follows. Section 2 presents related works. Section 3 illustrates the proposed cloud-based vehicular network architecture. Section 4 presents the real-time cloud based video capture service. In section 5, the proposed video capture service is implemented and evaluated. The conclusion is presented in Section 6.

\section{RELATED WORKS}

In [15], a hierarchical architecture for cloud-based vehicular networks is proposed that facilitates sharing of computational resources, storage resources and bandwidth resources among vehicles. The proposed architecture includes three types of 
cloud: a vehicular cloud, a roadside cloud, and a central cloud. This architecture does not consider the cellular cloud where many services and applications can be provided on cellular network. However, our architecture includes the cellular cloud.

In [16], Pics-on-Wheels service, a Vehicular Cloud service, is proposed which delivers images on demand to customers by using vehicles' on board cameras. The proposed service provides customer with images through internet server. The internet server assigns the photo shooting task to vehicles close to the target.

In [17], a comprehensive survey on vehicular cloud computing is presented that explore different area such as vehicular cloud architecture, application scenarios, security and privacy issues, key management strategies. The research paper gives a comprehensive taxonomy of vehicular networking and vehicular cloud computing. The services proposed for vehicular cloud computing are Network as a service (NaaS), Storage as a service (STaaS), Cooperation as a service (CaaS), Computing as a service, Pictures on a wheel as a service, Information as a service (INaaS) and entertainment as a service (ENaaS). In our research paper, a new service is proposed called video capture as a service.

An efficient digital video recording scheme for smart vehicle is proposed in [18]. The proposed scheme supports real-time video and data capture of vehicles in the intelligent transportation system. Our research work is an enhancement to [18] that utilized the cloud computing for vehicular networks.

Different from the aforementioned works, our research works present a new cloud based vehicular network architecture for ITS that it supports different applications and services. We proposed a new service called cloud based video capture. To the best of our knowledge, this is the first service to address real-time video capture of specific location, moving vehicle, and route in intelligent transportation system. Furthermore, new resource allocation and management algorithms are proposed to handle vehicle selection and video capture handoff from one vehicle to another with minimum service disruption time.

\section{THE PROPOSED CLOUD BASED VEHICULAR NETWORK ARCHITECTURE}

In this section, the proposed architecture is presented. It is depicted in figure 1 which enables a new cloud based ITS applications and services for road safety, traffic efficiency as well as for infotainment and business. It consists of four different types of cloud: vehicle cloud, roadside cloud, cellular cloud, central cloud. These types of cloud provide different services and it is accessed by different users such as vehicles, customers in internet and travellers. The different cloud can be used to share computation, storage, and bandwidth among different vehicles or users.

\subsection{Vehicular Cloud}

Vehicular cloud is local cloud established among a group of cooperative vehicles. These vehicles share storage, bandwidth, computing, and information. A group of collaborative vehicles can provide more widespread, consistent, and reliable services for the urban area where the single vehicle cannot.

The motivation for vehicular cloud is to share the computation, storage resources and real-time data capture among vehicles. The vehicular cloud can be utilized to share computation in some application such as In-Vehicle Multimedia Entertainment.

The implementation of vehicular cloud is very different from a traditional cloud in computer network. Vehicles in the vehicular cloud are dynamically changing due to mobility. We propose a new scheme for vehicular clouds implementation: Server Based (Centric) Vehicular Cloud Management (SBVCM). In this scheme, the vehicular cloud is centrally controlled and managed by server called cloud controller out of the vehicular cloud.

\subsection{Roadside Cloud}

A roadside cloud consists of dedicated servers attached to Roadside Units (RSUs). It is established among a set of adjacent roadside units. The dedicated servers virtualize physical resources and act as a potential cloud site. The RSUs provide the interface to access the servers. The roadside cloud can be accessed by vehicles and travellers through RSU or dedicated server in Internet.

The motivation for roadside cloud is to share the computation, storage resources and real-time data analysis and broadcast among vehicles and users. The roadside cloud can be utilized to provide services such as storage, computing, platform and other different services as in the traditional cloud. The difference between internet cloud and roadside cloud is in the services accessing by the moving vehicles. The implementation of roadside cloud can be handled using Virtual Machine (VM) to be accessed by vehicle or traveller [19].

\subsection{Cellular Cloud}

The cellular cloud is group of eNodes connected to different eNodes and gateways nodes. In this architecture we propose an enhancement to eNodes in order to support different application and services of the ITS.

The motivation for cellular cloud is to share the computation, storage resources and real-time data capture and analysis among vehicles and users. Cellular cloud is a local cloud driven by a set of adjacent eNodes. In a cellular cloud, there are dedicated local cloud servers attached to eNodes. A vehicle will access cellular cloud by V2I communications.

\subsection{Central Cloud}

The central cloud can be established among a set of dedicated servers in vehicular networks data center or servers in the Internet. The roadside cloud and the cellular cloud represent the network access layer.

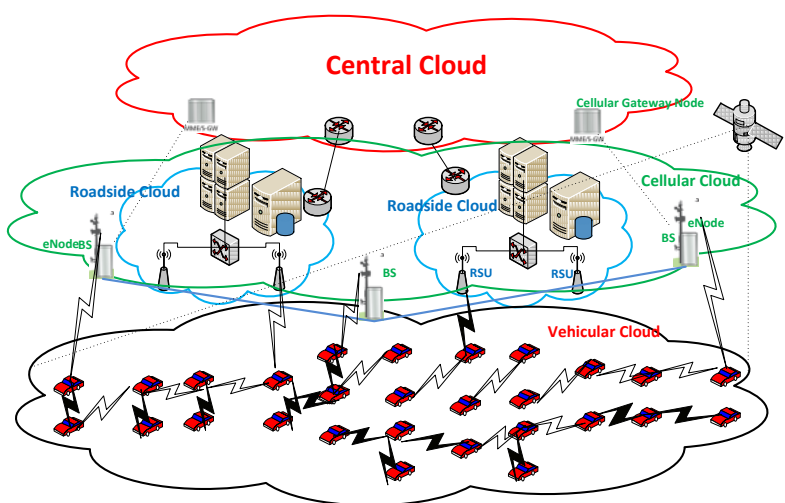

Figure 1: the proposed cloud based architecture 
From the above discussion, we suggest that a new system like WAVE should be proposed based on WAN network (WiMax 3G/LTE) with different function and services.

\section{THE PROPOSED REAL-TIME CLOUD BASED VIDEO CAPTURE}

In this section, a video capture service based on cloud computing is proposed. The vehicles can be utilized as cloud to capture real time video of specific location or moving vehicle. The video data is captured and transmitted to a server in Internet. Then, the video data is used when needed. The video data is retrieved using GPS coordination. Group of vehicles are controlled via dedicate server. The server can be private server or public server. The Server, vehicles and customers communicate via public network such as $3 \mathrm{G}$ or LTE or private network such as Wi-Fi or WAVE based network.

It is assumed that every vehicle has digital camera attached to the front of the vehicle. The vehicle moves on a road either in one direction, two way direction or cross circle. Any vehicle wants to provide video capture service should first register itself to server and then periodically announce its GPS location to the server. It is assumed that customer can request the service from internet for a specific location, a moving vehicle, or route on specific time. The specific location can be represented by either GPS location or name of location address from map.

We will use the following terminology which it is illustrated in Figure 2:

Vehicles Ready List (VRL): it is the list that contains all vehicles ready to provide service. The list has the fields: vehicle ID, current GPS location, and route.

Vehicles Service List (VSL): it contains the selected vehicles to provide the video capture service.

Zone of Service (ZOS): the area where vehicle is able to start video recording;

Cloud Controller (CC): The cloud service manager is responsible for resource allocation and video capture.

The video capture service is provided in two steps: vehicle registration and resources allocation and management. The vehicle registers with server that it is able to provide the service. The server accepts the request and it adds the vehicle to the VRL. The server sends acknowledgment to vehicle. The vehicle periodically updates its location by sending its GPS location.

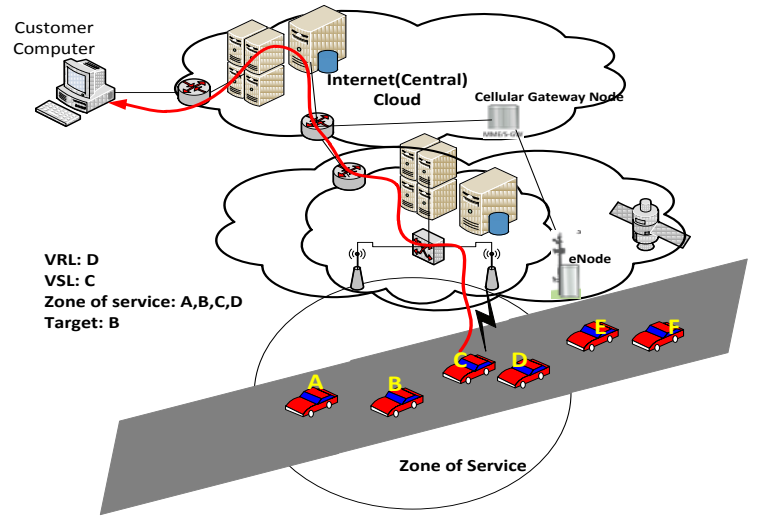

Figure 2: system model
The video capture service can be utilized in three different ways. The customer can request video capture service of specific location. When customer sends request to server to monitor the specified location, the request should include the GPS information of the location. The server invokes cloud controller to handle the video capture and resource management task. The cloud controller creates the two lists: VRL and VSL. The cloud controller uses the resource allocation and management algorithm to handle vehicles selection and video capture handoff. The cloud controller establishes connection with selected vehicles and the customer. The following algorithm shows the steps of resource allocation and management of specific location.

Algorithm 1: Resource Allocation (Video Capture of Specific Location)

Input: Target GPS location

BEGIN

For each vehicle update in the zone of Service.

\{

Compute Angle Deviation of the vehicle a angle_1

Compute Distance distance1

If (distance $1<\mathrm{d}$ OR angle_ $1>\theta$ ) Then

Stop video capture.

Remove from service list

Remove from Ready List.

//Select next candidate vehicle.

End if

"vehicle move in the direction of the target object

If(distance $1>$ d AND angle $1<\theta$ ) AND Not in Ready List Then

End if

Add to Ready list

If(distance1 $>$ d AND angle_1 $<\theta$ )AND Not in Service List AND Not in Ready List Then

Add to Service list

Establish connection

End if Start video capture.

\}

END

Another case of utilizing service is video capture of vehicle. The customer sends request for video capture of vehicle using its ID. The request contains the vehicle ID. The server forwards request to cloud controller. The cloud controller looks up the vehicle GPS location using its ID. If the vehicle ID is not found, the request is rejected. If the vehicle is in the cloud controller list, it invokes the resource allocation and management algorithm to handle vehicles selection and video capture handoff as in algorithm 2.

Algorithm 2: Resource Allocation (Video Capture of Moving Vehicle)

Input: Vehicle ID

BEGIN

For(each update of Vehicle ID )

\{

Compute current Zone of Service for Vehicle ID.

\}

For each vehicle update in the zone of Service)

Compute Angle Deviation of the vehicle angle_1

Compute Distance distance1

If (distance $1<\mathrm{d}$ OR angle_1 $>\theta$ ) Then

Stop video capture. 
Remove from service list

Remove from Ready List.

//Select next candidate vehicle.

End if

'vehicle move in the direction of the target object

If(distance1 $>$ d AND angle_1 $<\theta$ ) AND Not in Ready List Then

\section{End if}

Add to Ready list

If(distance1 $>$ d AND angle_1 $<\theta$ )AND Not in Service List AND Not in Ready List Then

Add to Service list

Establish connection

End if

Start video capture.

END

The customer sends request to server for video capture of full route. The request must contain the starting GPS point and the end GPS point. The vehicle that provide the service should send the route if the driver has selected a route. In this case, the cloud controller looks for vehicle with the same route. If the route exists, the cloud controller uses resource management of route as in algorithm 3 .

Algorithm 3: Resource Allocation (Video Capture of Route) Input: start GPS point, End GPS point.

BEGIN

Compute Zone of Service of start GPS point.

Compute route GPS points.

For each GPS point in the route

\{

Compute Zone of Service of next GPS point.

For each vehicle update in the zone of Service)

\{

Compute Angle Deviation of the vehicle angle_1

Compute Distance distance 1

If (distance $1<\mathrm{d}$ OR angle_1 $>\theta$ ) Then

Stop video capture.

Remove from service list

Remove from Ready List.

//Select next candidate vehicle.

End if

'vehicle move in the direction of the target object

If(distance $1>$ d AND angle $1<\theta$ ) AND Not in Ready List Then

End if

Add to Ready list

If(distance $1>\mathrm{d}$ AND angle_1< 1 )AND Not in Service List

AND Not in Ready List Then

Add to Service list

Establish connection

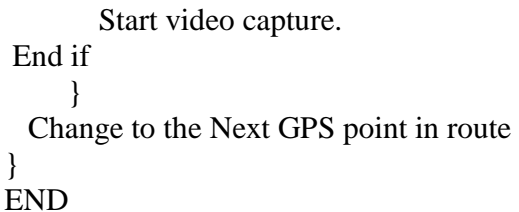

Suppose that we have the two GPS points (lat1dd, long1dd) and (lat2dd, long2dd). To compute the distance between two GPS points, the values of Latitude and Longitude should be in Decimal Degrees (dd.ddddd) in order to use the following Excel formula (1):

$=3963 *$ ACOS $(\operatorname{COS}($ RADIANS $(90$ -

lat1dd) $* \operatorname{COS}($ RADIANS(90-lat2dd))+SIN(RADIANS(90lat1dd) $*$ SIN(RADIANS(90-

lat2dd) $* \operatorname{COS}($ RADIANS(long1dd-long2dd))).

The result of the above formula is in miles. We can change miles value to meters by multiplying the value in miles by 1609.34. Suppose the target object point is (lat1dd,long1dd) and the vehicle location is (lat2dd,long2dd) as in figure 3 . When a vehicle update its point to (lat3dd,long3dd), we can compute the angle of deviation using triangle angle.

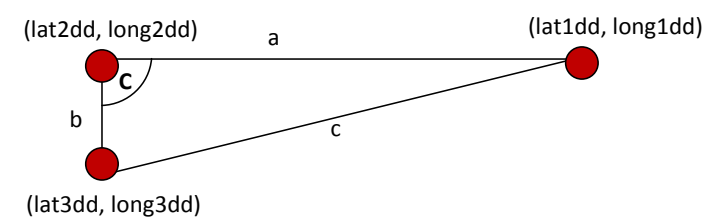

Figure 3: the angle of deviation.

The angle $\mathrm{C}$ of a triangle can be calculated from its sides using cosine law as in the following formula (2):

$\mathrm{C}=\cos ^{-1}\left(\left(\mathrm{a}^{2}+\mathrm{b}^{2}-\mathrm{c}^{2}\right) / 2 \mathrm{ab}\right)$

The above formulas in the first algorithm are studied using the Google maps [20] with map of figure 6. The scenario is to study the video capture service of a specific location. The specific location is $(15.278517,44.241651)$ that is sent by customer computer to server. The following tables are simple trace of the video capture handoff decision from one vehicle to another vehicle while monitoring the specified location. It is assumed that there are three vehicles A, B, and C.

Table 1 shows that vehicle A started providing service at distance 226 meters. The vehicle A moves towards the specified location $(15.278517,44.241651)$. When the vehicle distance is 179 meters from the specific location, the system make video capture handoff to vehicle B because the angle deviation is 73 degrees.

Table 1: Vehicle A Tracking during Service

\begin{tabular}{|c|c|c|c|c|c|c|}
\hline Update & Latitude & Longitude & Distance & $\begin{array}{l}\text { Angle } \\
\text { Deviation }\end{array}$ & Vehicle & State \\
\hline 1 & 15.276603 & 44.242359 & 226.2125569 & & A & in service \\
\hline 2 & 15.276701 & 44.242322 & 214.6030981 & 0.569778 & A & In \\
\hline 3 & 15.276856 & 44.242246 & 195.6201495 & 5.70146 & A & In \\
\hline 4 & 15.276975 & 44.242214 & 181.9811326 & 4.49736 & A & In \\
\hline 5 & 15.277032 & 44.242295 & 179.1829848 & 73.2919 & A & Handoff B \\
\hline
\end{tabular}


Table 2: Vehicle B Tracking during Service

\begin{tabular}{|c|c|c|c|c|c|c|}
\hline Update & Latitude & Longitude & Distance & Angle Deviation & Vehicle & State \\
\hline 1 & 15.276649 & 44.242345 & 220.8849597 & & B & $\begin{array}{l}\text { in } \\
\text { service }\end{array}$ \\
\hline 2 & 15.276783 & 44.242286 & 204.7081196 & 3.305852102 & $\mathrm{~B}$ & In \\
\hline 3 & 15.276908 & 44.242243 & 190.0506812 & 1.154780113 & B & In \\
\hline 4 & 15.277078 & 44.242173 & 169.7047188 & 2.108902672 & B & In \\
\hline 5 & 15.277172 & 44.242141 & 158.6936823 & 0.990853687 & B & In \\
\hline 6 & 15.277332 & 44.242071 & 139.4037233 & 3.512169082 & B & In \\
\hline 7 & 15.277518 & 44.241991 & 117.0424128 & 3.660933135 & B & In \\
\hline 8 & 15.277782 & 44.2419 & 86.07379162 & 0.204251732 & B & In \\
\hline 9 & 15.278046 & 44.241809 & 55.10547124 & 0.193792402 & B & In \\
\hline 10 & 15.278181 & 44.241766 & 39.38712404 & 0.850630732 & B & In \\
\hline 11 & 15.278201 & 44.241873 & 42.49177816 & 97.30569103 & B & Out \\
\hline
\end{tabular}

Table 2 shows that vehicle $\mathrm{B}$ started providing service at distance 220 meters. The vehicle B moves towards the specified location $(15.278517,44.241651)$. When the vehicle distance is 42 meters from the specific location, the system makes video capture handoff to vehicle $\mathrm{C}$ because the angle deviation is 97 degrees.

Table 3: Vehicle C Tracking during Service

\begin{tabular}{|r|l|r|r|r|l|l|}
\hline \multicolumn{1}{|c|}{ Update } & \multicolumn{1}{l|}{ Latitude } & \multicolumn{1}{|c|}{ Longitude } & Distance(m) & Angle Deviation & Vehicle & state \\
\hline 1 & 15.276783 & 44.242275 & 204.3177144 & & $\mathrm{C}$ & in service \\
\hline 2 & 15.276814 & 44.242275 & 201.0610103 & 19.12144386 & $\mathrm{C}$ & In \\
\hline 3 & 15.277099 & 44.242168 & 167.3212043 & 0.467732447 & $\mathrm{C}$ & In \\
\hline 4 & 15.277228 & 44.24212 & 152.0650631 & 0.541606638 & $\mathrm{C}$ & In \\
\hline 5 & 15.277498 & 44.242007 & 119.6971937 & 2.6449288 & $\mathrm{C}$ & In \\
\hline 6 & 15.277673 & 44.241943 & 99.04302556 & 0.814346188 & $\mathrm{C}$ & In \\
\hline 7 & 15.277979 & 44.241835 & 63.06186736 & 0.349236593 & $\mathrm{C}$ & In \\
\hline 8 & 15.278175 & 44.241766 & 40.02190823 & 0.490504878 & $\mathrm{C}$ & In \\
\hline 9 & 15.278346 & 44.241712 & 20.13031945 & 1.042365705 & $\mathrm{C}$ & In \\
\hline 10 & 15.278491 & 44.241658 & 2.988753767 & 0.697391958 & $\mathrm{C}$ & In \\
\hline 11 & 15.278513 & 44.241658 & 0.871027033 & 14.51571781 & $\mathrm{C}$ & Out \\
\hline
\end{tabular}

Table 3 shows that vehicle $\mathrm{C}$ started providing service at distance 204 meters. The vehicle $\mathrm{C}$ moves towards the specified location $(15.278517,44.241651)$. When the vehicle distance is 0.8 meter from the specific location, the system vehicle $\mathrm{C}$ becomes out of service because the distance is smaller than one meter.

The problem in the above resource allocation is either the large number of vehicles in the zone of Service needs high bandwidth network to transfer the captured video or no vehicle in the zone of service. The network load makes network slow. If the server records all the traffic, it need hug storage. The solution to this problem is to use one source of data capture in each direction. If there are many vehicles in zone of video capture, the server should compute for each vehicle the following: 1-Direction 2-distance 3- Deviation of the vehicle. The server selects the best candidate vehicle which has the best distance and angle deviation of the vehicle.

Another problem in the above solution is the delay associated with video capture handoff from one vehicle to another. The handoff delay is the round trip time between server and new candidate vehicle. We propose a video capture handoff scheme with minimum delay and minimum disruption time. To solve this problem, the server establishes connection with the two best candidate vehicles and forward video of one of them to customer. Then server periodically checks the distance and angle deviation of the two vehicles. The server replaces any vehicle if it is not satisfy video capture requirement.

Figure 4(a) shows the video capture of moving vehicle A before the handoff. We can see that vehicle B is in the VSL. 
Vehicle $C$ and Vehicle D are in the VRL. Figure 4(b) shows that vehicle $\mathrm{B}$ turned right with angle deviation greater than $\theta$. In this case, the $\mathrm{CC}$ adds the vehicle $\mathrm{C}$ to the VSL. It establishes connection with Vehicle C. Then, Vehicle C starts video capture service.

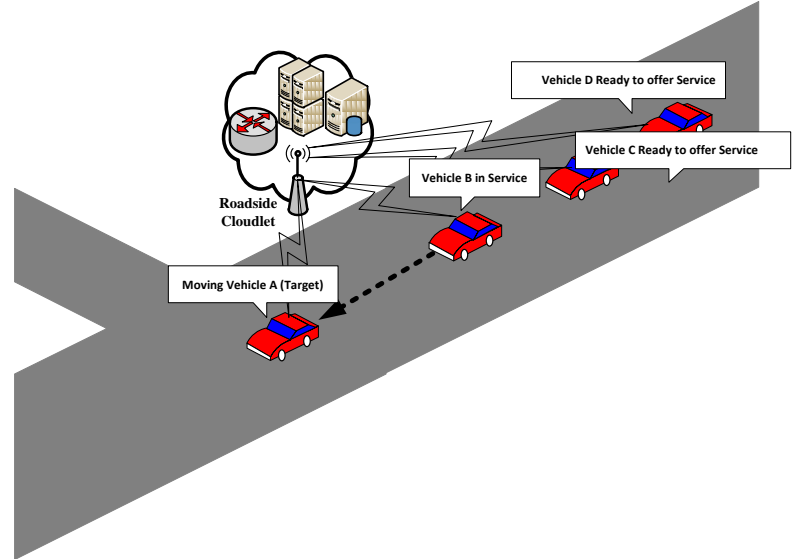

Figure 4(a): video capture handoff (Before handoff)

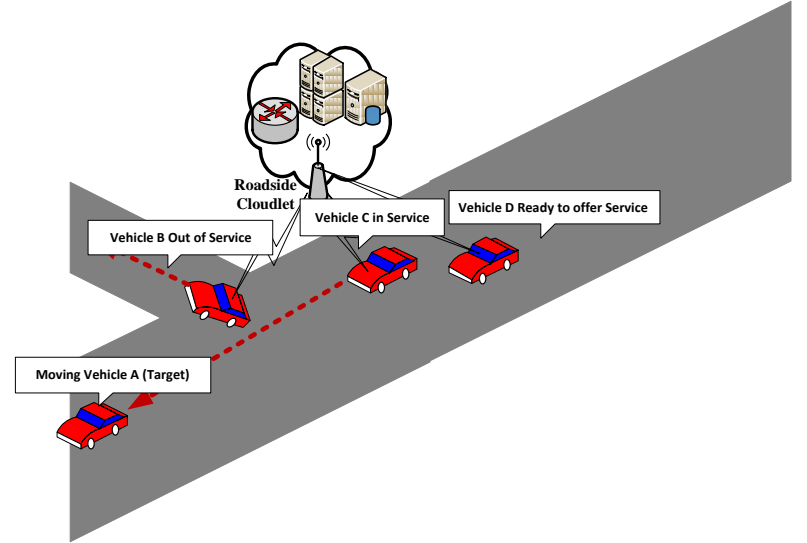

Figure 4(b): video capture handoff (After handoff)

\section{SYSTEM IMPLEMENTATION}

The proposed system can be studied and evaluated using testbed implementation. Figure 5 shows a logical view of how all the components interact with each other while providing a framework for video capture service studying. The testbed model consists of 4 cars. Each car has its digital camera, computer system and GPS device (Mobile Android with digital camera). The 4 cars are connected to $\mathrm{WiFi}$ wireless LAN with outdoor access point. The access point is connected to the server. The server is connected with computer using Ethernet LAN. The system is distributed on the car computer system, server, and customer computer. The area of service is shown in the map of figure 6 . It is the road around the Alandalus University. The zone of service is 250 meters from the target Object. We use Android SDK 2.3.3 / API 10 to code the mobile system [21,22]. Microsoft SQL server is used for creating database at server.

The implemented system needs further work to enable us to study its performance. The application in the android mobile gets GPS information and sends it periodically to the server. In the same time this application sends real time video data to the server based on server request. The SBVCM scheme proposed in section 3.1 is used to control the vehicular cloud. Further work is required to study the service disruption time during video capture handoff from one vehicle to another. The delay and number of packet loss should be computed in order to show the performance of the proposed service.

\section{CONCLUSION}

In this article, a cloud-based architecture for vehicular networks that facilitates sharing of computational resources, storage resources and bandwidth resources among vehicles is presented. Then, we focused on real-time cloud based video capture in the proposed architecture. The resource allocation algorithm is proposed to manage vehicle selection in order to provide video capture service. A novel approach for video capture handoff algorithm is proposed to change service from one vehicle to another with minimum service disruption and delay. Finally, the proposed video capture handoff is evaluated and discussed.

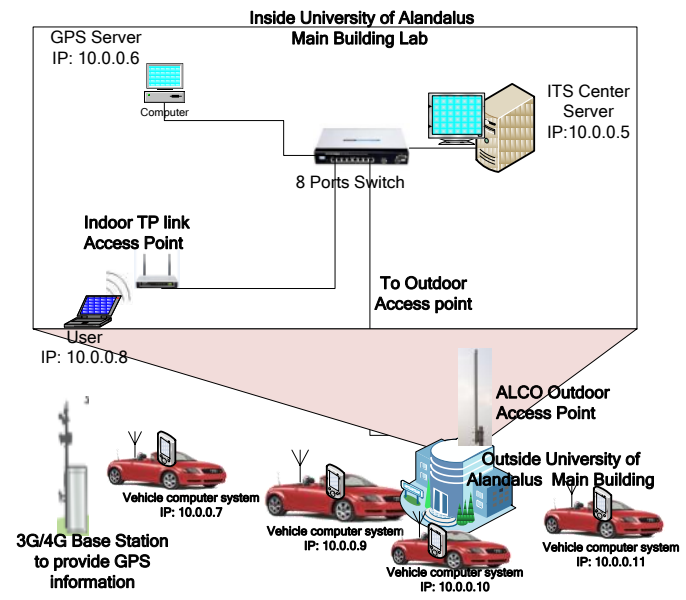

Figure 5: system Test bed and components

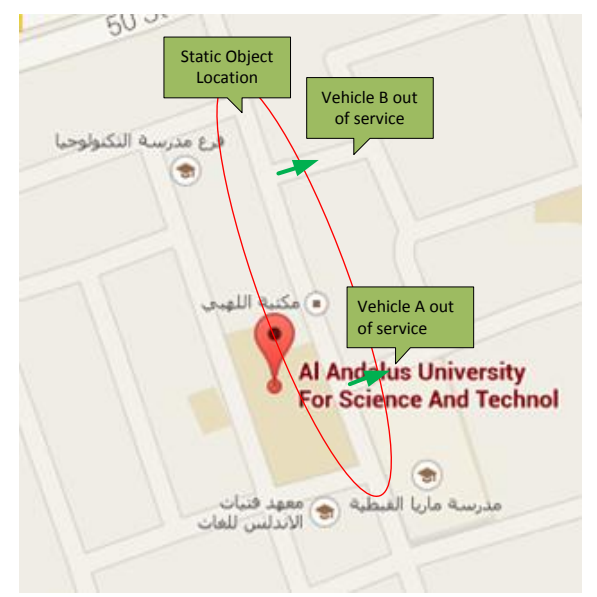

Fig. 6: Map of Video Capture Zone

\section{ACKNOWLEDGMENTS}

A substantial part of this research was financed by Alandalus University, Republic of Yemen. I am grateful to University of Alandalus for having believed that this might be a real and reasonable investment.

\section{REFERENCES}

[1] Research and Innovative Technology Administration (RITA), U.S. Department of Transportation (US DOT) 1200 New Jersey Avenue, http:/ /www.its.dot.gov /data_capture/ data_capture.htm, last time accessed 5/3/ 2013. 
[2] ERTICO Intelligent Transport Systems and Services stakeholders in Europe: http://www.ertico.com/ertico-itseurope/, last accessed on June 10th, 2014.

[3] Ministry of Land, Infrastructure, Transport and Tourism in Japan: http://www.mlit.go.jp/en/index.html, last accessed on June 10th, 2014.

[4] Intelligent Transportation Systems Society of Canada: http://www.itscanada.ca, last accessed on June 10th, 2014

[5] Connie Ribeiro, Bringing Wireless Access to the Automobile: A Comparison of Wi-Fi, WiMAX, MBWA, and $3 \mathrm{G}, 21 \mathrm{st}$ Computer Science Seminar, http://citeseerx.ist.psu.edu/viewdoc/summary?doi=10.1.1 .149 .9385 .

[6] Sherali Zeadally, Ray Hunt, Yuh-Shyan Chen, Angela Irwin, Aamir Hassan, Vehicular ad hoc networks (VANETS): status, results and challenges, Springer Science+Business Media, LLC 2010.

[7] Gerlach, M. (2006). Full paper: assessing and improving privacy in VANETs. www.network-onwheels.de/downloads/escar2006gerlach.pdf (accessed: May 29, 2010).

[8] Jiang, D., Delgrossi, L., IEEE 802.11p: Towards an International Standard for Wireless Access in Vehicular Environments, IEEE Vehicular Technology Conference, 2008. VTC Spring 2008., May 2008, , pages: 2036 2040 .

[9] Yunpeng Zang, Erik Weiss, Lothar Stibor, Bernhard Walke, Hui Chen, Xi Cheng, Opportunistic Wireless Internet Access in Vehicular Environments Using Enhanced WAVE Devices, International Journal of Hybrid Information Technology, 2008.

[10] Yougui Liu; Baoxing Bai; Research on GPRS vehicle location network service system, International Conference on Computer, Mechatronics, Control and Electronic Engineering (CMCE), Changchun,china 2010.

[11] European Telecommunications Standards Institute 2010, ETSI TS 102 636-3 V1.1.1, Intelligent Transport Systems (ITS); Vehicular Communications; GeoNetworking; Part 3: Network architecture, Technical Specification, (2010-03), http://www.etsi.org

[12] S. Olariu, M. Eltoweissy and M. Younis, "Towards Autonomous Vehicular Clouds," ICST Transactions on
Mobile Communications and Applications, vol. 11, no. 7-9, pp. 1-11, 2011.

[13] D. Bernstein, N. Vidovic and S. Modi, "A Cloud PAAS for High Scale, Function, and Velocity Mobile Applications - With Reference Application as the Fully Connected Car, in Proc. Fifth International Conference on Systems and Networks Communications (ICSNC), pp. 117-123, 2010.

[14] R. Hussain, J. Son, H. Eun, S. Kim and H. Oh, "Rethinking Vehicular Communications: Merging VANET with cloud computing", in Proc. IEEE 4th International Conference on Cloud Computing Technology and Science, pp. 606-609, 2012.

[15] Rong Yu, Yan Zhang, Stein Gjessing, Wenlong Xia, Kun Yang, Toward Cloud-based Vehicular Networks with Efficient Resource Management, IEEE Network, (Volume:27 ,Issue: 5 ), Oct 2013, pp. 48 - 55.

[16] M. Gerla, J.-T. Weng, and G. Pau, "Pics-on-wheels: Photo surveillance in the vehicular cloud," in Computing, Networking and Communications (ICNC), 2013 International Conference on. IEEE, 2013, pp. 11231127.

[17] Md Whaiduzzaman , Mehdi Sookhak, Abdullah Gani, Rajkumar Buyya, A survey on vehicular cloud computing, Journal of Network and Computer Applications, Elsevier, 2013.

[18] Fekri M. Abduljalil, Ali Alsharafi," An Efficient Digital Video Recording Scheme for Intelligent Transportation Systems", World Congress on Computer and Information Technology ( WCCIT'2013), IEEE Computer Society, Sousse, Tunisia, June 22-24, 2013.

[19] M. Satyanarayanan, P. Bahl, R. Caceres, and N. Davies. "The Case for VM-based Cloudlets in Mobile Computing", IEEE Pervasive Computing, vol. 8, no. 4, 2009.

[20] google maps, https://www.google.com/maps/preview, last time accessed 1/6/2014

[21] Android SDK, Eclipse + ADT plugin, http://developer.android.com/sdk/index.html, last time accessed 20/06/2014.

[22] Java SE Development Kit 7, http://www.oracle.com/technetwork/java/javase/downloa ds/jdk7-downloads-1880260.html, last time accessed on 20/06/2014. 